\title{
X-ray evaluation of the Marshall Grazing Incidence X-ray Spectrometer (MaGIXS) nickel-replicated mirrors
}

\author{
Patrick Champey $^{\mathrm{a}}$, Amy R. Winebarger ${ }^{\mathrm{a}}$, P.S. Athiray ${ }^{\mathrm{b}}$, Ken Kobayashi ${ }^{\mathrm{a}}$, Sabrina Savage ${ }^{\mathrm{a}}$, \\ Jeffery K. Kolodziejczak ${ }^{\mathrm{a}}$, Jacqueline Davis ${ }^{\mathrm{a}}$, Charlie Griffith ${ }^{\mathrm{a}}$, Edward N. Hertz ${ }^{\mathrm{c}}$, Peter \\ Cheimets $^{\mathrm{c}}$, and Brian D. Ramsey ${ }^{\mathrm{a}}$ \\ ${ }^{a}$ NASA Marshall Space Flight Center, Huntsville, AL 35812 \\ bNASA Postdoctoral Program, NASA Marshall Space Flight Center, Huntsville, AL 35812 \\ 'Smithsonian Astrophysical Observatory, 60 Garden St., Cambridge, MA 02138
}

\begin{abstract}
X-ray observations of astronomical objects provides diagnostics not available in any other wavelength regime, however the capability of making these observation at a high spatial resolution has proven challenging. Recently, NASA Marshall Space Flight Center (MSFC) has made good progress in employing computer numerical control $(\mathrm{CNC})$ polishing techniques on electroless nickel mandrels as part of our replicated grazing incidence optics program. CNC polishing has afforded the ability to deterministically refine mandrel figure, thereby improving mirror performance. The Marshall Grazing Incidence X-ray Spectrometer (MaGIXS) is a MSFC-led sounding rocket instrument that is designed to make the first ever soft x-ray spectral observations of the Sun spatially resolved along a narrow slit. MaGIXS incorporates some of the first mirrors produced at MSFC using this polishing technique. Here we present the predicted mirror performance obtained from metrology, after completion of CNC polishing, as well as the results of X-ray tests performed on the MaGIXS telescope mirror before and after mounting.
\end{abstract}

Keywords: X-ray Spectrometer, Soft X-ray, Solar, Sounding Rocket, Replicated Optics, Deterministic Polishing

\section{INTRODUCTION}

One of the most frequently studied phenomena in solar physics is the heating of the solar corona, where the temperature increases from $6000 \mathrm{~K}$ at the photosphere, or the surface of the Sun, to $>1 \mathrm{MK}$ in the corona. Significant theoretical and observational progress has been made in unraveling this puzzle, and several hypotheses about the mechanisms driving coronal heating. Currently two models, magnetic reconnection and wave dissipation, lead the debate over how hot plasma is heated. However, despite more than three decades of continuous observations of the sun from the visible to X-ray wavelengths, a significant gap exists in spectral measurements throughout the soft X-ray. Specifically, Ref. 1 reported a blind spot in the differential emission measure (DEM) of high-temperature, low emission measure plasma, where this observational coverage is lacking (Figure: 1a). The slope of the high temperature DEM fall-off above $5 \mathrm{MK}$ is not well constrained; this slope can be used to determine the frequency of heating and further constrain the heating mechanism. ${ }^{2}$ The Marshall Grazing Incidence X-ray Spectrometer (MaGIXS) is an instrument that is designed to observe, for the first time, soft $\mathrm{X}$-ray spectra of high-temperature, low-emission plasma of coronal structures spatially resolved along a narrow slit. Measurements from these observation will extend the DEM coverage from 3MK to 10MK, which will help constrain the slope of the DEM fall-off. MaGIXS is a NASA sounding rocket experiment designed to observe soft X-ray emission from $0.5-2.0 \mathrm{keV}$ energies $(24-6.0 \AA)$ in an active region core. The the spectral lines shown in Figure: $1 \mathrm{~b}$ are the dominant lines that populate this band. The key emission lines are Fe XVII, Fe XVIII, and Fe XIX. Measuring the line ratios of these species will provide a good measure of the DEM at temperatures extending beyond $5 \mathrm{MK}$.

Further author information: (Send correspondence to P. Champey)

P. Champey: E-mail: patrick.r.champey@nasa.gov, Telephone: 256.961.2268 


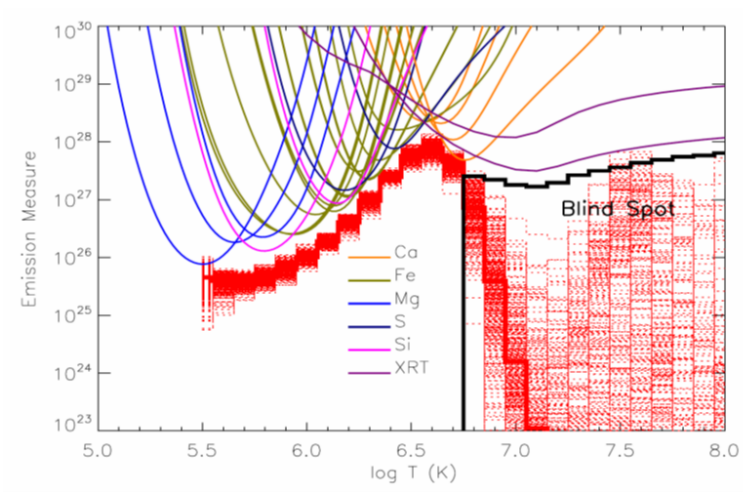

(a)

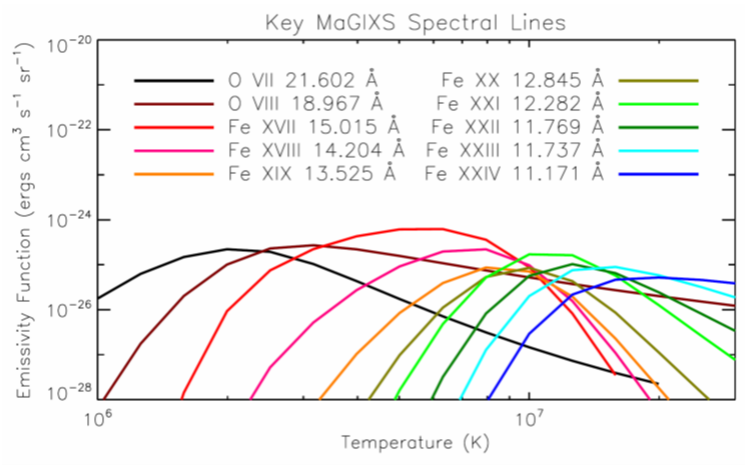

(b)

Figure 1: Left panel: The Extreme-ultraviolet Imaging Spectrometer (EIS) and X-Ray Telescope (XRT), both instruments on Hinode, have a "blind-spot" in temperature/emission measure space. They are not sensitive to plasma with temperature greater than $\sim 6 \mathrm{MK}$ and line-of-sight emission measures less than $10^{27} \mathrm{~cm}^{-5}$. Right panel: The emissivity functions of the key MaGIXS spectral lines. The MaGIXS wavelength ranges includes strong lines formed from 2 to $>10 \mathrm{MK}$, including lines from all ionization stages of from Fe XVII to XXIV.

\section{MAGIXS INSTRUMENT}

The MaGIXS instrument has been under development for more than 10 years at NASA MSFC and partner institutions, the Smithsonian Astrophysical Observatory (SAO), the Massachusetts Institute of Technology (MIT), and Izentis LLC. As the lead institution, The Marshall Space Flight Center (MSFC) has developed the replicated mirrors, optical bench, detector and avionics, and will provide launch support. SAO has designed and fabricated the telescope mirror assembly (TMA) and the spectrometer optical assembly (SOA), which included precision mounting and alignment of the mirrors within those assemblies. ${ }^{3}$ MIT and Izentis LLC. have designed and fabricated the silicon grating. Their technique utilizes the $\{111\}$ crystal plane, which is cut so that it is aligned with the blaze angle. The grating lines are printed using e-beam lithography, and then they are etched. Currently the TMA and SOA are being integrated to the optical bench, and co-aligned at the MSFC X-ray and Cryogenic Facility (XRCF). MaGIXS is scheduled to launch from White Sands Missile Range (WSMR), New Mexico in the spring of 2020.

The MaGIXS optical design is shown in Figure 2. MaGIXS comprises a single shell, nickel-replicated Wolter-I telescope with a slit jaw (context imager), and a soft X-ray spectrometer. An 8 arcminute long, 5 arcsecond wide slit is placed at the focal plane of the telescope. On the back-side of the slit is a finite conjugate mirror pair, a blazed, varied-line space reflective grating and a CCD detector. The finite conjugate mirror pair (SM1 and SM2 respectively) are single parabola mirrors replicated from a single mandrel. Between SM2 and the detector sits a reflective grating that will disperse the converging (focused) cone of rays to the detector, positioned off axis. The MSFC developed CCD detector will detect the first-order diffraction. ${ }^{4,5}$ The stigmatic optical design gives good performance on and off-axis, out to a 4 arcminute field angle. The desired performance across the full field is 6 arcsecond angular resolution, and $\sim 22 \mathrm{~m} \AA(7.5-0.47 \mathrm{eV})$ spectral resolution. ${ }^{3,6-8}$

Although this unique optical design provides good spatial and spectral performance, there were several challenges associated with mirror development and alignment. The mirror prescriptions for MaGIXS have large cone angles, and a moderate amount of sag (aspheric deviation from conical taper), which had an impact on metrology accuracy, and reduced lap polishing efficiency. From an alignment perspective, the alignment tolerances generated from the optical error budget were very tight. Unlike coaxially nested telescope systems, MaGIXS has three grazing incidence mirrors aligned and focused serially, with the largest separation spanning $\approx 1.5$ meters. The system is most sensitive to alignment error between the two spectrometer mirrors, which require 5" co-alignment. ${ }^{3}$ Currently, both the TMA and SOA have been assembled with internal alignment well within requirements. At MSFC, the instrument is being integrated, with alignment and focusing between the TMA and SOA ongoing. We anticipate the system to complete alignment in fall 2019, and undergo end-to-end wavelength calibration in early 2020 . 


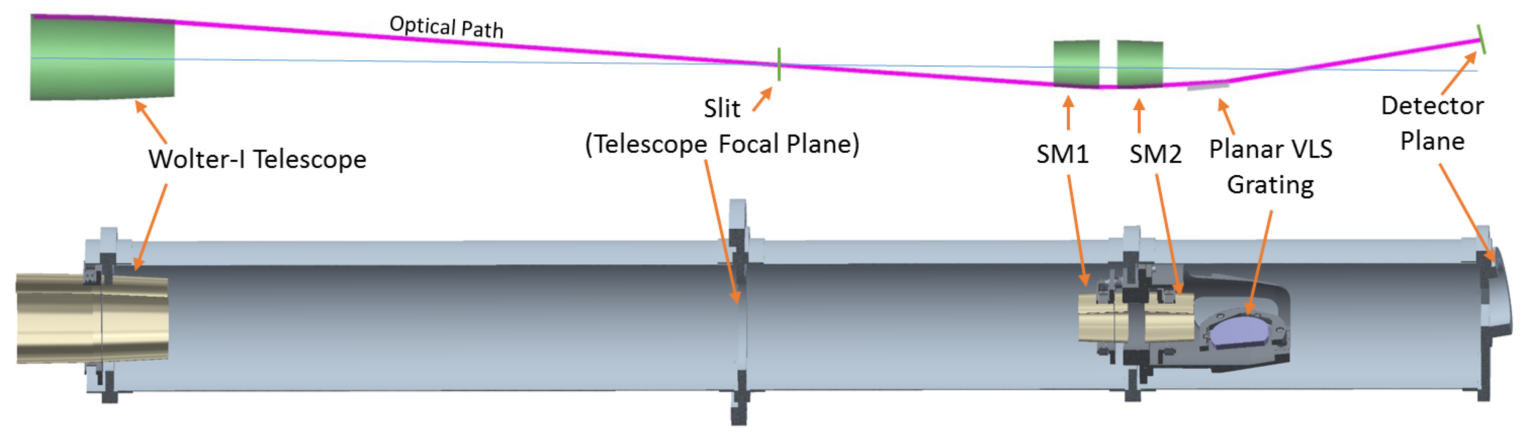

Figure 2: A ray trace of the MaGIXS optical design is shown above the solid model of the MaGIXS instrument. All major optical components are listed. Not shown are the slit jaw imaging system, scientific detector, and entrance and focal plane filters.

\section{MIRROR FABRICATION}

The MaGIXS mirrors were produced using MSFC's electro-forming replication process - the same process used to produce HEROES,,${ }^{9,10}$ ART-XC, ${ }^{11-14}$ FOXSI,,${ }^{15-18}$ and IXPE. ${ }^{19,20,20}$ Fabrication begins with rough cutting a mandrel from an aluminum block. A conical taper is cut into the mandrel, then the mandrel is plated with electroless nickel (EN). This hard EN over coat is diamond turned, generating the mirror's figure. After diamond turning, the mandrel undergoes several polishing iterations to remove residual diamond turning tool marks, and bring the surface quality to within $\leq 5 \AA$ surface roughness. At this point the mandrel is replicated in a nickelcobalt bath, and a thin shell optic is produced. The shell is separated from the mandrel in a chilled water bath, completing the process. Depending on the application, the inner optical surface of these shells can be coated to enhance reflectively and increase effective area.

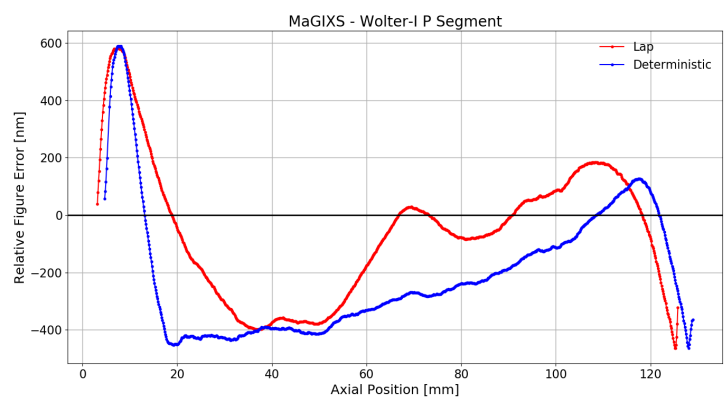

(a) Figure error of paraboloid segment.

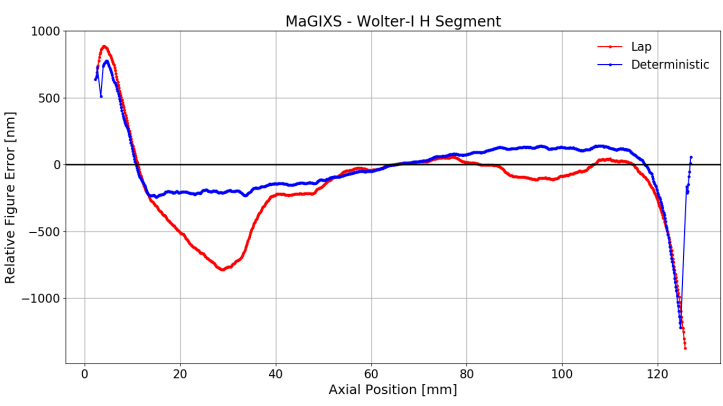

(b) Figure error of hyperboloid segment.

Figure 3: Axial figure error profiles of the $\mathrm{P}$ (left) and $\mathrm{H}$ (right) segments of the Wolter-I telescope mandrel. This data is the raw output obtained using an interferometer with only a transmission flat - the data have not been re-scaled, or processed to compensate for measurement errors. In both panels, the red curves represent the figure after diamond turning and sevelap polishing, while the blue curves represent the figure after the mandrel figures were deterministically refined using the Zeeko IRP $600 X \mathrm{CNC}$ polisher. A significant reduction in low-spatial-frequency figure errors was observed, specifically for spatial wavelengths $>7 \mathrm{~mm}$. There are midspatial-frequency errors still present below this spatial wavelength range that are not sensitive to the bonnet tools used for this polishing effort.

In most cases, the quality of the diamond turned figure may be sufficient for meeting the figure requirements. After diamond turning, the mandrels are sent directly into lap polishing, which brings the surface roughness into specification. During the lap polishing phase, it is common to observe a reduction in mid-spatial frequency 
figure error, as well as the surface roughness, but an increase in low-frequency error. It is typical to see mandrels finishing with about 10 - 15 arcsecond predicted half-power-diameter (HPD), and surface roughness $\leq 5 \AA$, with replicated mirror shells roughly two times worse in performance. While this approach is robust, and straight forward to implement, it is not deterministic, and therefore is not ideal for refining figure down to the arcsecondlevel.

In the case for MaGIXS, a highly deterministic polishing technique was employed with the goal to reduce slope errors to $\approx 1.5$ ", or better. The MSFC developed processes utilizes a Zeeko IRP $600 X$ computer-numericalcontrol (CNC) polishing machine, which we have demonstrated is capable of polishing electroless nickel-coated mandrels using a series of bonnet-style tools. After several polishing passes, a significant reduction in slope errors was observed on both mandrel figures for spatial wavelengths $>7 \mathrm{~mm}$. Figure 3 shows the figure error for the paraboloid and hyperboloid segments of the Wolter-I telesceope. The red curves are from axial profile measurements taken after lap polishing, while the blue curves were measured after several iterations of deterministic polishing. The large deviations at the ends of the curves are physical edges that we avoid while polishing; these features provide alignment fiducials when comparing metrology from different polishing runs. Therefore, considering only the corrected regions, the axial slope errors were reduced to 0.72 arcseconds on the P segment, and 1.26 arcseconds on the $\mathrm{H}$ segment. If we consider both the axial and azimuthal components, the combined RMS slope error is 1.0" and 1.55" on the $\mathrm{P}$ and $\mathrm{H}$ segments, respectively. From these values the estimated HPDs are 2.71" and 4.19", for $\mathrm{P}$ and $\mathrm{H}$ respectively, and 4.99" for $\mathrm{P}+\mathrm{H}$ combined. If we then consider the edge effects, the estimated HPDs become 3.54", 5.34" and 6.41", respectively. For HPD estimation, we consider the slope errors to follow a Gaussian distribution, where the RMS slope error is $\approx \sigma$. We then consider the half-power radius of a Gaussian distribution to be 1.35 , so the HPD becomes $2.7 \sigma$.

During this polishing effort, just 100 degrees of the full 360 degree revolution was corrected. This was done intentionally to fit within project schedule, and was afforded by the fact that the planar grating sets the usable aperture to $\approx 36$ degrees. Additionally, due to limitations imposed by metrology, we focused only on reducing the axial slope errors - i.e. we did not address figure errors in the azimuthal direction. We observed significant variations in metrology scaling that was trivial to correct for in the axial component, but did not converge as well in the azimuthal component. Before we can begin to confidently reduce these azimuthal errors, we need to improve our measurement accuracy. Ongoing work is currently addressing these metrology shortcomings, which should lend the ability to address other components of figure error. ${ }^{21}$

One caveat to replication is that replicated shells end up with worse performance than what is predicted for metrology performed on the mandrel. It is understood that mirror shells will not exactly replicate the mandrel surface. Historically, replication errors degrade the performance by about a factor of two, as compared to the mandrel. This is largely due to imparted stresses that are generated during the replication and shell release process. The sources of these imparted errors, namely low-frequency figure errors, that tend to occur near the mirror edges, are not yet well understood. Currently at MSFC, research is underway to identify and address the sources of such errors, in an attempt to reduce, or control imparted stresses during replication.

\section{X-RAY EVALUATION: ON-AXIS PERFORMANCE}

The MaGIXS mirrors were tested in the Stray Light Facility (SLF) at MSFC using the 100 meter beam line. A set of Wolter-I mirrors and single paraboloid mirrors were tested before deterministic polishing was employed, and then several finished shells were tested before delivery to SAO for mounting. Here we present the results of the X-ray tests performed on the mounted Wolter-I (i.e. the TMA) and the two spectrometer mirrors before they were mounted. For each test, the mirrors were installed onto the 3-axis stage (pitch, yaw and focus), which sits inside a 24 inch diameter vacuum bell jar. An Andor Ikon-L $2 \mathrm{kX} 2 \mathrm{k}$ CCD detector was positioned at the end of the bell jar, at nominal focal plane of the mirror. Figure 4 is a collage of the testing setup inside the bell jar where the optics are mounted. For the TMA test a retractable aperture blocker was used to isolate the sub-aperture that will be dispersed by the grating. The blocker was placed on a retractable stage so that alignment and focusing could be done first with the full aperture, which is much more intuitive with a circular symmetry.

For each mirror test, several sets of images were taken at different positions on either side of best focus. These through-focus PSF measurements provide information that allow us to evaluate the on-axis response of the optic, 


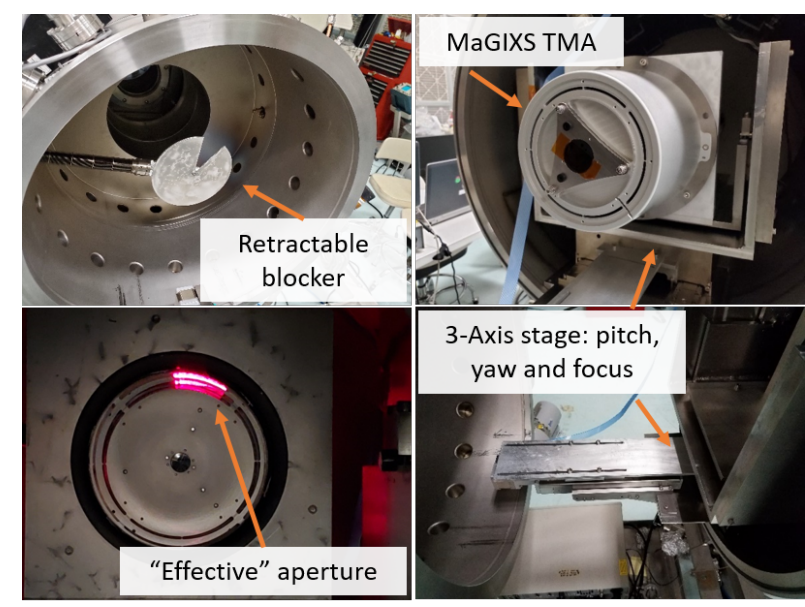

Figure 4: Testing configuration in the SLF at the MSFC. Top Left: The aperture masked installed to a retractable linear stage, placed in front of the TMA. Top Right: The MaGIXS TMA mounted to the 3-axis stage inside of the vacuum bell jar. Bottom Left: An image of the rear of the TMA aperture, with the blocker in place. Note the alignment laser is entirely eclipsed by the mask, except for the selected sub-aperture. Bottom Right: The three axis stage, which provides fine pitch, yaw and focus adjustments for alignment and focusing in vacuum.

and quantify characteristics such as angular resolution, depth of focus, azimuthal errors (i.e. circularity) and scattering - though we only discuss angular resolution and focusing in this paper. We captured images at \pm 6 $\mathrm{mm}$ down to $\pm 1 \mathrm{~mm}$ at $1 \mathrm{~mm}$ step size, and then increased resolution to $0.1 \mathrm{~mm}$ from $+0.9 \mathrm{~mm}$ to $-0.9 \mathrm{~mm}$. Prior to these scans, we first had to find best focus by minimizing the HPD using pitch, yaw and focusing adjustments. Referencing this position on the focusing stage encoder, we were able to precisely drive to the positions on either side of focus.

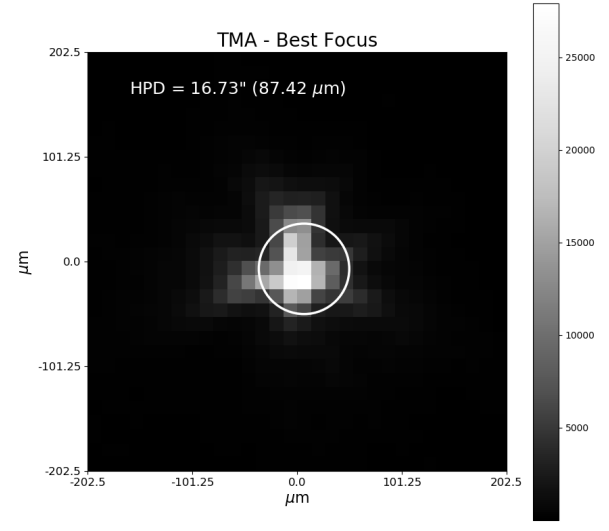

(a) Full aperture.

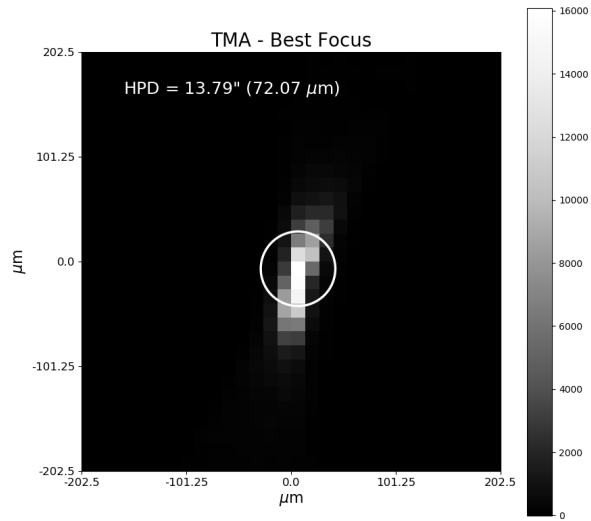

(b) Sub-aperture.

Figure 5: TMA on-axis PSFs for the full aperture (left), and sub-aperture(right). For the full aperture case, the HPD is 16.7" $(87 \mu \mathrm{m})$, and wings of the PSF show the result of aberrations caused by the non-circularity features. For the sub-aperture, the HPD of the spot is $13.8 "(72 \mu \mathrm{m})$.

Figure 5a is an image of the PSF of the full TMA aperture. The measured HPD is 16.7" $(87 \mu \mathrm{m})$, while the full-width half-max (FWHM) computed from the $2 \mathrm{D}$ Gaussian fit is $\approx 10$ " in the $\mathrm{X}$ and Y directions. Note that 


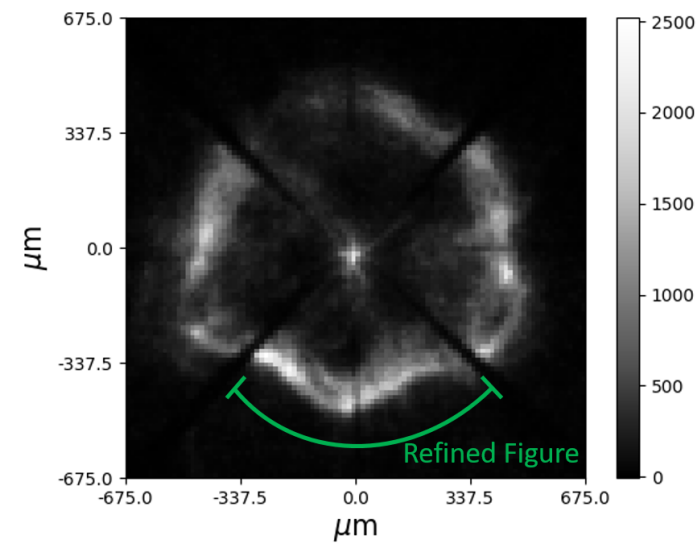

(a) $6 \mathrm{~mm}$ intra-focus

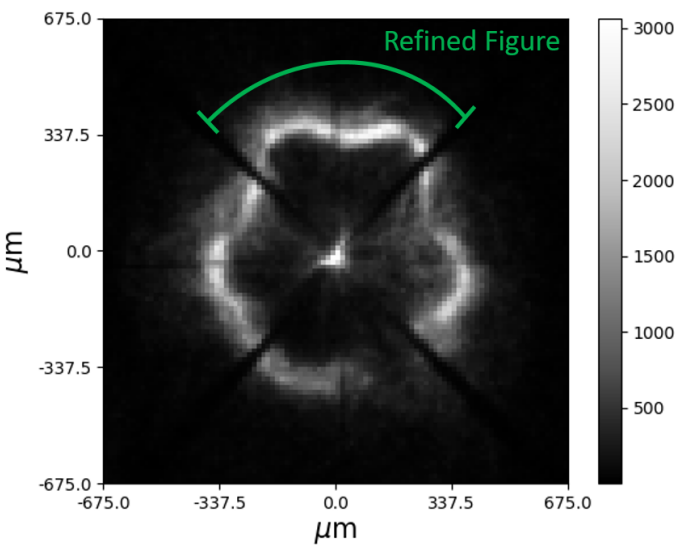

(b) $6 \mathrm{~mm}$ extra-focus

Figure 6: Defocused images of the TMA. These images provide insight to several mirror characteristics, specifically sources of aberration caused by azimuthal errors in the mirror shell, the figure quality which is related to the width of the annulus, and the scattering.

the PSF has distinct features toward the wings, which is an effect of the geometric aberrations generated from the azimuthal errors in the mirror. The shape of the mirror aperture can be seen in Figure 6. With the blocker inserted in front of the TMA, the PSF generated by the segment of the aperture dispersed by the grating could then be imaged and characterized directly. Figure 5b shows the best focus spot of the dispersed aperture, which has a characteristic bow tie shape typical of segmented grazing incidence mirrors. The HPD of the best focused spot is 13.8" $(72 \mu \mathrm{m})$. In the case for MaGIXS, the narrow width of the bow tie lies in the dispersion direction of the grating, while the broader width is in the cross dispersion direction. The cross dispersion direction contains the imaging component of spectrometer - i.e. this direction of the PSF lies along the length of the slit.

Figure 6 shows a pair of defocused images at $6 \mathrm{~mm}$ inside (6a) and outside of focus (6b), with the deterministically polished region of the mirror annotated in both images. This sub aperture appears to produce a cleaner response. It can be seen that the annulus in this region is narrower, has a higher intensity, and generates less scattering than the other regions of the annulus. Fitting a Gaussian function to the cross section of the annuls produced by the refined regions reveals an RMS HPD of $\sim 13$ ", which is consistent with the sub-apertured PSF in Figure 5b. We are working to further characterize the scattering component in these images, which should give a better measure of the figure quality, represented by the annulus. Nevertheless, these results are encouraging because it suggests that refining mandrel figure does propagate through the replication process. A similar response is observed for the spectrometer mirrors, where the refined regions of the mirror produce a cleaner response than other portions of the aperture. After the instrument is fully integrated, aligned and focused, we will complete a similar end-to-end through focus test to character the system and directly measure the resolution.

The images in Figure 6 also reveal significant aberration that is a direct result of azimuthal error in the mirror. It is common to observe low-order azimuthal error that manifests as an oval, or ellipse, which generates astigmatism. Mounting replicated optics can also cause these types of azimuthal errors, but we ruled out mounting effects, as X-ray tests were performed prior to mounting the mirror. The data show the same features were present just after replication. In general, the higher-order azimuthal error observed here is not typical of replicated mirrors. In this particular case, it is likely the result of damage incurred on the mandrel during fabrication. The mandrel impacted a sharp edge, and was subsequently repaired. We believe the mandrel has a similar azimuthal figure error that has not been detected in interferometric metrology, simply due to undersampling the azimuthal component of the figure. We plan to follow up with contact profilometry that we believe can detect and quantify this type of error. It must also be noted that the azimuthal errors described as a result of this incident are not the same as the errors described in Section 3.

Through-focus images can be used to determine the location of best focus, and also depth of focus. By evaluating the annulus at several positions on either side of focus, the optimal focal position is found using interpolation. We measured the HPD of each image, with the center of the PSF, or annulus for defocused PSFs, 
as the origin. We then fit a pair of lines to the HPD as a function of relative position, for each side of focus. Figure 7 is a plot of the HPD as a function of defocus distance, for both the full aperture case (green), and the sub-aperture case (red). We found that in both cases, the depth of focus approximately $\pm 0.5 \mathrm{~mm}$ from the best focus position. We used this depth of focus knowledge to successfully focus the TMA on the plane of the slit during focusing tests in the XRCF at MSFC. We recorded sets of data with the TMA focused to three different positions, and from those measurements determine the correct spacer length for the TMA interface to the optical bench. A fourth set of images were captured, and we were able to confirm TMA focus.

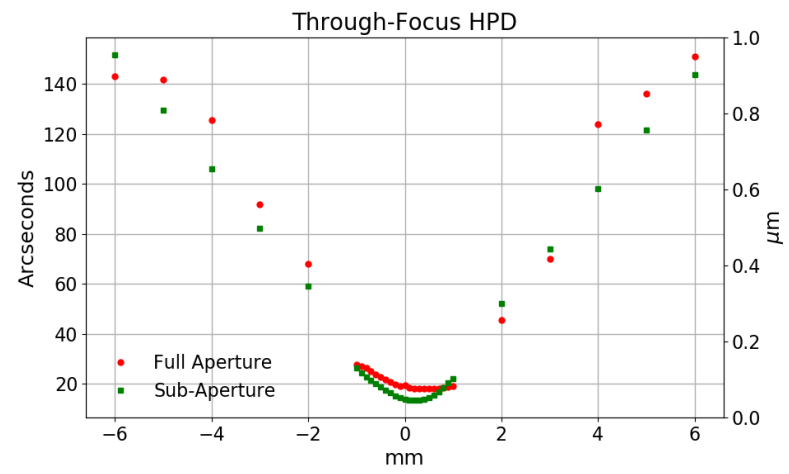

Figure 7: HPD over the focal position, relative to best focus. The red points were measured from full-aperture images, while the green points were measured from sub-aperture images. The depth of focus in both cases is \pm $0.5 \mathrm{~mm}$, which meant that we could relax the focusing requirement for TMA.

Like the Wolter-I mandrel, a 100 degree region of the spectrometer mandrel was refined with the deterministic polishing technique. X-ray test of the shells replicated from the spectrometer mirror mandrels were performed and the HPDs were measured; however, images are not included here. The measured full aperture HPDs were 21.1" and 19.3", for SM1 and SM2 respectively. The out-of-focus images show the mirrors have subtle low-order azimuthal error, which does produce an observable astigmatism at best focus, but not nearly as severe as the TMA. For these tests, the aperture blocker was not implemented, so we do not have a direct measure of the sub-aperture PSF. However, using this data, we were able to predict the on-axis, end-to-end HPD (minus the grating). We generated an image mask that represented the slit filed stop at the TMA focal plane, and multiplied it with Figure 5a. We then took the convolution of the two spectrometer PSFs, and convolved the result with the slit-masked TMA PSF. The product (Figure 8) was a symmetric PSF, with an HPD of $\sim 31$ ". This result includes the full aperture of each of the three mirrors, so we take this as the upper-limit of the MaGIXS PSF. We also compared this result to the quadrature sum of the measured HPDs reported above, which yielded 31.5". We expect the PSF produced by the deterministically polished sub-aperture to be smaller. Nevertheless, this upper limit PSF is within expectation for replicated optics. Historically, the angular resolution of replicated systems with nested configurations is between 20-30" ${ }^{9,14,18}$

\section{CONCLUSION}

MaGIXS comprises a Wolter-I telescope and a finite conjugate pair of single parabolid replicated mirrors, a blazed varied line space grating, and an MSFC develop CCD detector with sounding rocket flight heritage. The tight toleranced optical design presented challenges in mirror fabrication and alignment, which were addressed by employing state-of-the-art polishing techniques and developing a visible-light alignment approach. The polishing technique yielded significantly improved mandrel figure, as observed in metrology. However, the X-ray test demonstrated marginal improvement in measured HPD, likely the product of replication imparted stresses, and aberrations generated from higher-order azimuthal errors in the mirror. The measured HPD of the TMA subaperture is 13.8". The predicted upper-limit on-axis performance of the Wolter-I and the two spectrometer mirrors is $\approx 31$ " - this includes a larger fraction of the aperture that did not have the figure refined. Despite this larger than predicted PSF, the performance falls within the expectations for replicated optics, in this type 


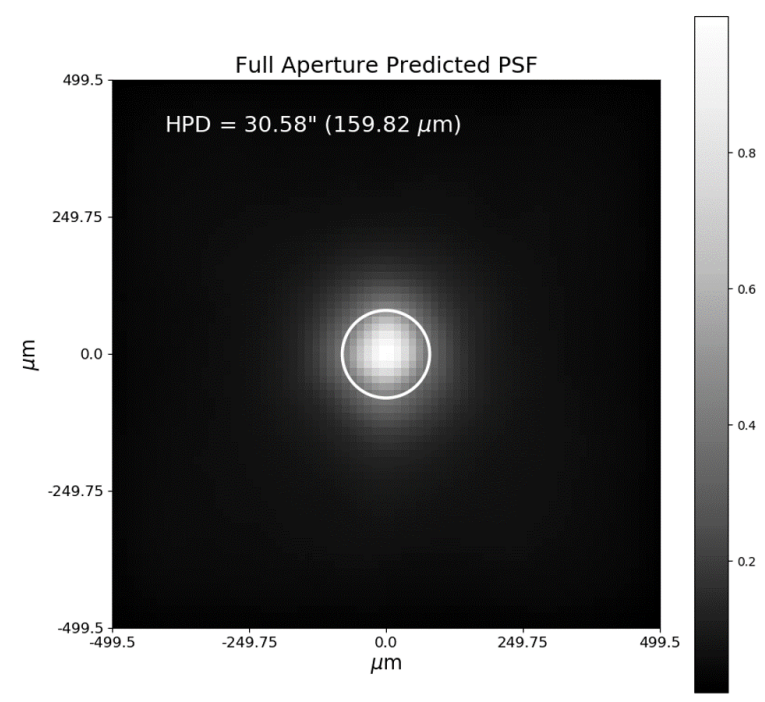

Figure 8: The predicted n-axis PSF of the TMA, SM1 and SM2, not including the grating. This image was generated by taking the TMA PSF projected onto a slit, and convolving the image with the spectrometer images. The result is a symmetric PSF, with a 31" HPD.

of arrangement. The current status of MaGIXS is the TMA has been integrated to the optical bench, and has been focused in X-rays at MSFC in the XRCF. The TMA and SOA are being co-alignment and focusing using visible light, and once complete will be tested end-to-end in the XRCF. Instrument integration and alignment is expected to be completed in fall 2019, with end-to-end wavelength calibration in early 2020.

\section{ACKNOWLEDGMENTS}

The MaGIXS instrument team is supported by NASA Low Cost Access to Space program. P.S. Athiray's research is supported by an appointment to the NASA Postdoctoral Program at the Marshall Space Flight Center, administrated by Universities Space Research Association under contract with NASA.

\section{REFERENCES}

[1] Winebarger, A. R., Warren, H. P., Schmelz, J. T., Cirtain, J., Mulu-Moore, F., Golub, L., and Kobayashi, K., "Defining the "Blind Spot" of Hinode EIS and XRT Temperature Measurements," 746, L17 (Feb. 2012).

[2] Athiray, P. S., Winebarger, A. R., Barnes, W. T., Bradshaw, S. J., Savage, S., Warren, H. P., Kobayashi, K., Champey, P., Golub, L., and Glesener, L., "Solar Active Region Heating Diagnostics from High Temperature Emission using the Marshall Grazing Incidence X-ray Spectrometer (MaGIXS)," arXiv e-prints , arXiv:1909.02541 (Sep 2019).

[3] Champey, P., Winebarger, A., Kobayashi, K., Savage, S., Cirtain, J., Cheimets, P., Hertz, E., Golub, L., Ramsey, B., McCracken, J., Marquez, V., Allured, R., Heilmann, R. K., Schattenburg, M., and Bruccoleri, A., "On the alignment and focusing of the Marshall Grazing Incidence X-ray Spectrometer (MaGIXS)," in [Space Telescopes and Instrumentation 2016: Ultraviolet to Gamma Ray], Proc. SPIE 9905, 990573 (July 2016).

[4] Champey, P., Kobayashi, K., Winebarger, A., Cirtain, J., Hyde, D., Robertson, B., Beabout, D., Beabout, B., and Stewart, M., "Performance characterization of UV science cameras developed for the Chromospheric Lyman-Alpha Spectro-Polarimeter (CLASP)," Proc. SPIE 9144, 914439-914439-7 (2014).

[5] Champey, P., Kobayashi, K., Winebarger, A., Cirtain, J., Hyde, D., Robertson, B., Beabout, D., Beabout, B., and Stewart, M., "VUV testing of science cameras at MSFC: QE measurement of the CLASP flight cameras," Proc. SPIE 9601, 9601 - 9601 - 5 (2015). 
[6] Kobayashi, K., Cirtain, J., Golub, L., Korreck, K., Cheimets, P., Hertz, E., and Caldwell, D., "Stigmatic grazing-incidence x-ray spectrograph for solar coronal observations," in [Space Telescopes and Instrumentation 2010: Ultraviolet to Gamma Ray], Proc. SPIE 7732, 773233 (July 2010).

[7] Kobayashi, K., Cirtain, J., Golub, L., Winebarger, A., Hertz, E., Cheimets, P., Caldwell, D., Korreck, K., Robinson, B., Reardon, P., Kester, T., Griffith, C., and Young, M., "The Marshall Grazing Incidence X-ray Spectrograph (MaGIXS)," in [Optics for EUV, X-Ray, and Gamma-Ray Astronomy V], Proc. SPIE 8147, 81471M (Oct. 2011).

[8] Kobayashi, K., Winebarger, A. R., Savage, S., Champey, P., Cheimets, P. N., Hertz, E., Bruccoleri, A. R., Scholvin, J., Golub, L., Ramsey, B., Ranganathan, J., Marquez, V., Allured, R., Parker, T., Heilmann, R. K., and Schattenburg, M. L., "The Marshall Grazing Incidence X-ray Spectrometer (MaGIXS)," in [Space Telescopes and Instrumentation 2018: Ultraviolet to Gamma Ray], Proc. SPIE 10699, 1069927 (July 2018).

[9] Ramsey, B. D., Alexander, C. D., Apple, J. A., Benson, C. M., Dietz, K. L., Elsner, R. F., Engelhaupt, D. E., Ghosh, K., Kolodziejczak, J. J., O'Dell, Stephen L.and Speegle, C. O., Swartz, D. A., and Weisskopf, M. C., "HERO: program status and first images from a balloon-borne focusing hard x-ray telescope," in [X-Ray Optics for Astronomy: Telescopes, Multilayers, Spectrometers, and Missions], Proc. SPIE 4496, $4496-4496-6$ (2002).

[10] Christe, S. D., Shih, A., Rodriguez, M., Cramer, A., Kyle, G., Edgerton, M., Gaskin, J., Wilson-Hodge, C., Jeff, A., Katherine, S., Jackson, A., Smith, L., Dietz, K., Brian, O., Sobey, A., Koehler, H., and Ramsey, B. D., "The high energy replicated optics to explore the sun mission: a hard x-ray balloon-borne telescope," in [Solar Physics and Space Weather Instrumentation V], Proc. SPIE 8862, 8862 - 8862 - 13 (2013).

[11] Gubarev, M., Ramsey, B., O’Dell, S. L., Elsner, R., Kilaru, K., McCracken, J., Pavlinsky, M., Tkachenko, A., Lapshov, I., Atkins, C., and Zavlin, V., "Development of mirror modules for the ART-XC instrument aboard the Spectrum-Roentgen-Gamma mission," in [Optics for EUV, X-Ray, and Gamma-Ray Astronomy VI], Proc. SPIE 8861, 88610K (Sept. 2013).

[12] Gubarev, M., Ramsey, B., Kolodziejczak, J. J., O’Dell, S. L., Elsner, R., Zavlin, V., Swartz, D., Pavlinsky, M., Tkachenko, A., and Lapshov, I., "The calibration of flight mirror modules for the ART-XC instrument on board the SRG mission," in [Space Telescopes and Instrumentation 2014: Ultraviolet to Gamma Ray], Proc. SPIE 9144, 91444U (July 2014).

[13] Gubarev, M., Ramsey, B., Elsner, R. F., O’Dell, S., Kolodziejczak, J., McCracken, J., Zavlin, V., Swartz, D., Kilaru, K., Atkins, C., Pavlinsky, M., Tkachenko, A., and Lapshov, I., "ART-XC/SRG: status of the x-ray optics development," in [Space Telescopes and Instrumentation 2014: Ultraviolet to Gamma Ray], Proc. SPIE 9144, 91441V-91441V-6 (2014).

[14] Krivonos, R., Tkachenko, A., Burenin, R., Filippova, E., Lapshov, I., Mereminskiy, I., Molkov, S., Pavlinsky, M., Sazonov, S., Gubarev, M., Kolodziejczak, J., O’Dell, S. L., Swartz, D., Zavlin, V. E., and Ramsey, B. D., "Calibration of the ART-XC mirror modules at MSFC," Experimental Astronomy 44, 147-164 (Oct 2017).

[15] Krucker, S., Christe, S., Glesener, L., McBride, S., Turin, P., Glaser, D., Saint-Hilaire, P., Delory, G., Lin, R. P., Gubarev, M., Ramsey, B., Terada, Y., Ishikawa, S.-n., Kokubun, M., Saito, S., Takahashi, T., Watanabe, S., Nakazawa, K., Tajima, H., Masuda, S., Minoshima, T., and Shomojo, M., "The Focusing Optics X-ray Solar Imager (FOXSI)," in [Optics for EUV, X-Ray, and Gamma-Ray Astronomy IV], Proc. SPIE 7437, 743705-743705-10 (2009).

[16] Krucker, S., Christe, S., Glesener, L., Ishikawa, S.-n., McBride, S., Glaser, D., Turin, P., Lin, R. P., Gubarev, M., Ramsey, B., Saito, S., Tanaka, Y., Takahashi, T., Watanabe, S., Tanaka, T., Tajima, H., and Masuda, S., "The Focusing Optics X-ray Solar Imager (FOXSI)," Proc. SPIE 8147, 814705-814705-14 (2011).

[17] Krucker, S., Christe, S., Glesener, L., Ishikawa, S., Ramsey, B., Gubarev, M., Saito, S., Takahashi, T., Watanabe, S., Tajima, H., Tanaka, T., Turin, P., Glaser, D., Fermin, J., and Lin, R. P., "The focusing optics x-ray solar imager (FOXSI): instrument and first flight," in [Solar Physics and Space Weather Instrumentation V], Proc. SPIE 8862, 88620R-88620R-12 (2013).

[18] Christe, S., Glesener, L., Buitrago-Casas, C., Ishikawa, S.-N., Ramsey, B., Gubarev, M., Kilaru, K., Kolodziejczak, J. J., Watanabe, S., Takahashi, T., Tajima, H., Turin, P., Shourt, V., Foster, N., and Krucker, S., "Foxsi-2: Upgrades of the focusing optics x-ray solar imager for its second flight," Journal of Astronomical Instrumentation 05(01), 1640005 (2016). 
[19] Weisskopf, M. C., Baldini, L., Bellazini, R., Brez, A., Costa, E., Dissly, R., Elsner, R. F., Fabiani, S., Matt, G., Minuti, M., Muleri, F., O'Dell, S., Pinchera, M., Ramsey, B., Rubini, A., Sgro', C., Soffitta, P., and Spandre, G., "A small mission featuring an imaging x-ray polarimeter with high sensitivity," in $[U V, X$ Ray, and Gamma-Ray Space Instrumentation for Astronomy XVIII], Proc. SPIE 8859, 885908-885908-15 (2013).

[20] Weisskopf, M. C., Ramsey, B., O’Dell, S., Tennant, A., Elsner, R., Soffitta, P., Bellazzini, R., Costa, Enrico andKolodziejczak, J., Kaspi, V., Muleri, F., Marshall, H., Matt, G., and Romani, R., "The Imaging X-ray Polarimetry Explorer (IXPE)," in [Space Telescopes and Instrumentation 2016: Ultraviolet to Gamma Ray], Proc. SPIE 9905, 990517-990517-10 (2016).

[21] Christe, S. D., Shih, A., Rodriguez, M., Cramer, A., Kyle, G., Edgerton, M., Gaskin, J., Wilson-Hodge, C., Jeff, A., Katherine, S., Jackson, A., Smith, L., Dietz, K., Brian, O., Sobey, A., Koehler, H., and Ramsey, B. D., "Deterministic polishing of replicating grazing-incidence mandrels," in [Optics for EUV, X-Ray, and Gamma-Ray Astronomy IX], Proc. SPIE 11119, 111190U (2019). 